\title{
«Expertos» en Bioética
}

\author{
M. TERESA LÓPEZ DE LA VIEJA \\ Universidad de Salamanca
}

\begin{abstract}
«Será también tarea de los bioéticos regular el crecimiento de la población mundial, reducirlo en la práctica. Otra vez estamos en la encrucijada. Sólo por ello habrá que preguntarse qué parte de patrimonio genético debe fomentarse en el sentido de la bioética y cuál puede incluso eliminarse. Todo ello requiere soluciones y planificación a largo plazo» ${ }^{1}$.
\end{abstract}

Resumen. ¿Es correcto hablar de «bioéticos»? Por una parte, la Bioética es una de las disciplinas más conocidas en Ética. Por otra, no se puede hablar en sentido estricto de «bioéticos», a tenor de su difuso papel. El artículo defiende que la disciplina exige conocimientos especializados. Pero un papel borroso para los «expertos» en Bioética, en Ética aplicada: 1) El experto en Filosofía moral corresponde mejor al papel restringido, modesto, que han de asumir los profesionales en tal campo. Éstos tendrán «competencia epistémica», no «competencia preformativa» en cuestiones prácticas. 2) Un debate fluido entre expertos procedentes de varios campos demostraría que es del todo innecesaria la figura de un «experto» en Bioética, o «bioético». 3) Pues todos los ciudadanos pueden valorar las cuestiones prácticas. Como agentes morales.

Hasta hace poco era infrecuente que alguien, ajeno a los temas de Ética aplicada o de Ética médica, se ocupase de «bioética» o de «bioéticos». La situación ha cambiado de forma apreciable en los últimos años. El escritor y Premio Nobel en Literatura G. Grass se ha referido con ironía a las crecientes expectativas, así como a los oscuros temores que suscitan las nuevas
AbStRact. What does the term «bioethicist» mean? On the one hand, Bioethics is becoming a very well-know discipline in Ethics. On the other hand, we could not talk properly about «bioethicists». Since their role is weakly defined. This article argues in favor of specialized knowledge in Bioethics. But it claims a fuzzy role for the «experts» in Bioethics, and in applied Ethics: 1) The expert in Moral Philosophy would be more suitable to the limited, moderate role that professionals have to take for granted there. They could be «epistemic competent», they could not claim the "performative competence» in practical issues, though. 2) Experts from many fields looking for open arguments in these topics would show that «experts in Bioethics», «bioethicist» are actually inessential for the discipline. 3) Having moral competence, citizens are able of valuing by themselves practical issues.

tecnologías en la opinión pública. En su obra Mi siglo, un científico envía una carta a alguien conocido, tal vez otro experto como él, a fin de comentarle en detalle su reciente viaje y su participación en un congreso mundial sobre embriología. De paso, intenta tranquilizarle sobre los posibles efectos que se derivarían de la clonación de seres humanos, justificando 
incluso las ventajas que dicha técnica podría aportar a las futuras generaciones. En el relato aparecen los términos en cuestión, «bioética» $\mathrm{y}$ «bioéticos». Este ultimo es usado como sinónimo de «expertos», expertos en Ética médica, en Bioética. De forma habitual se les conoce también como «especialistas en moral» ${ }^{2}$. En la carta, los bioéticos son mencionados como la máxima autoridad en temas de clonación, patrimonio genético, crecimiento demográfico, etc. Científicos, especialistas que, algún día, llevarán a los humanos más allá de lo conocido, y más allá de caducas ideas morales... La ficción sitúa al lector ante un futuro inquietante, que hoy provoca distintas reacciones y, en todo caso, está dando lugar a fuertes controversias. El relato es llamativo porque refleja las dudas más comunes sobre las técnicas aplicadas a la especie humana. Y, también, porque el texto de G. Grass normaliza términos de perfiles borrosos: «bioético», experto en Bioética.

¿Es correcto hablar de «bioéticos» en el mismo sentido en que hablamos de «Bioética»? La disciplina desarrolló de forma espectacular entre finales de los años sesenta y comienzos de los setenta. Extensión de la Ética o «Ética basada en conocimiento biológico» ${ }^{3}$ —según la definición del Dr. Potter van Rensselaer-, la nueva teoría intentaba actuar como un puente. El puente que necesitaban tanto el conocimiento científico como, de otra manera, los valores morales. La nueva especialidad creció en importancia, consolidándose pronto en el ámbito académico ${ }^{4}$. Poco después, los medios no especializados y la opinión pública se interesaban vivamente por los problemas y por los avances de esta disciplina, tendencia que ha continuado hasta hoy. Puede decirse incluso que la Bioética se ha convertido a lo largo de las últimas décadas en la parte más visible, la más conocida de la Ética. Es a veces la única disciplina ética que salta los muros académicos. Mucho menos clara resulta, sin embargo, la figura de aquellos profesionales que se ocupan de las cuestiones propias de la Bioética. ¿Puede decirse que un conocedor de la Literatura es un escritor? ¿Un experto en Filosofía es un «filósofo»? De forma análoga, iquien se dedica a temas de Bioética ha de tener un estatuto profesional como «bioético»? A pesar de que en determinados contextos exista la necesidad - e incluso la demanda- de personas o de grupos que sean competentes en temas de moralidad, a pesar también de que los profesionales en Ciencias de la salud e investigadores en Biología diferencian claramente entre aspectos técnicos y aspectos morales de sus respectivas prácticas, a pesar de todo ello, las demandas no crean ni justifican una práctica totalmente nueva, que sólo correspondería a los «bioéticos». ¿Quiénes son, qué papel les está reservado?

Da la impresión de que la respuesta dependerá de la forma de entender la Bioética como teoría. Se trata, sin duda, de una disciplina. Sin embargo, antes que nada es un ámbito de descubrimiento y de debate. Un «discurso» ${ }^{5}$, como prefiere llamarlo A. R. Jonsen. Quienes se dedican a la Filosofía - a la Filosofía prácticaestán en las mejores condiciones para analizar las cuestiones de moralidad, debido a su trabajo intensivo sobre argumentos y textos filosóficos. Por este motivo, tienen - o deberían tener- un lugar reservado entre quienes se interesan y quienes elaboran propuestas en los distintos campos de la Ética. Pero no un lugar exclusivo. Esto mismo vale para médicos y otros profesionales. La competencia moral no es exclusiva de quienes se dedican a una actividad, por mucho que ésta suscite dilemas morales. Ni está bajo la autoridad de quienes tienen un contacto más directo con las situaciones reales, médicos, juristas, psicólogos, etc. La competencia moral pertenece a todos los agentes. Por eso resulta conveniente que los «expertos» tengan un perfil difuso. La forma en que están aquí 
regulados los Comités asistenciales de Ética —regulados por parte de la Administración central y por parte de las Administraciones autonómicas - demuestra que, efectivamente, los temas de Bioética y de Ética médica requieren de un trabajo interdisciplinario. Como contrapartida, la Bioética no pertenece en exclusiva a los profesionales de la ciencia, de la Medicina o de la Filosofía. No pertenece a un tipo de expertos, puesto que la disciplina fue pensada para tender puentes entre campos. La Bioética invitan al aprendizaje mutuo. Requiere, en fin, de una gran cantidad de expertos en campos muy diversos, médicos, técnicos sanitarios, juristas, psicólogos, trabajadores sociales, administradores, economistas, filósofos... No de un «experto» dedicado de manera exclusiva a las cuestiones morales.

El artículo suscribe la tesis de que la Bioética es un «discurso»y, por lo mismo, acepta las pretensiones reducidas de los profesionales dedicados a estos temas: 1) La figura del experto en Filosofía moral, ayuda a compaginar dos elementos en tensión: las numerosas demandas que han surgido en torno a la Bioética y, de otro lado, el papel restringido, modesto, que han de asumir quienes se ocupan de la disciplina. Sean éstos médicos, juristas, psicólogos, sociólogos, economistas, representantes de las instituciones, psicólogos, educadores, filósofos, etc. De ahí procede el papel débil o restringido del filósofo moral en las situaciones prácticas. Es decir, puede mostrar las ventajas de la «competencia epistémica», no un nivel superior de «competencia preformativa» o práctica en la solución de los problemas. 2) Por tanto, la Bioética, como disciplina ética, cumple un papel difícilmente reemplazable a la hora de evaluar las nuevas técnicas, su impacto y sus riesgos. La perspectiva de argumentos compartidos, así como un debate fluido entre expertos procedentes de varios campos -interesados por igual en cuestiones morales-, hacen del todo innecesaria la figura de un «experto» en Bioética, o «bioético». 3) Éstos han de dar cuenta de sus argumentos y decisiones ante los ciudadanos, pese o precisamente porque éstos suelen tener un nivel modesto de información. Todos podrán valorar los planteamientos generales y las decisiones. Como agentes morales que son.

\section{EXPERTOS EN FILOSOFÍA MORAL}

«El buen sentido es la cosa mejor repartida del mundo, pues cada uno piensa que está tan bien provisto de ella que aquellos más difíciles de contentar en cualquier otra cosa no desean más de aquel que ya tienen» ${ }^{6}$. R. Descartes ironizaba sobre el universal uso de la razón. De manera más desdibujada, se refirió más adelante a las reglas de la moralidad. Éstas conformaban una «Moral provisional» ${ }^{7}$, que subrayaba la distancia entre el ámbito del conocimiento y el de la acción. Aquella manera provisional o abierta de enfrentarse a la moralidad ha sido luego positiva para el desarrollo de las distintas teorías morales. Sin embargo, puede haber sido también la causa remota de una imagen débil, que todavía pesa sobre la reflexión moral. Incluso hoy es poco visible la actividad de los filósofos, pese a que éstos participan ocasionalmente en el debate público, en las instituciones, como especialista junto a otros especialistas, etc. La hipótesis que aquí se defiende: no es imprescindible el experto en Bioética, sí lo es el trabajo de expertos con varia formación para abordar las cuestiones morales de forma competente. Estos profesionales aportarán distintos enfoques sobre los dilemas morales, sin que ello afecte a la competencia profesional de los filósofos. Al contrario. Éstos, los profesionales de la Filosofía, utilizan y aportan información altamente especializada sobre tales cuestiones. Sin embargo, ello no parece motivo bastante 
como para crear una nueva figura, el «bioético» o especialista en cuestiones de Bioética. Sólo en Bioética. Así pues, la escasa visibilidad social de los filósofos - los motivos que han producido esta situación han sido y son diversos - puede justificar su presencia, activa, especializada, en la esfera académica y en la esfera pública. Pero no un papel distinto. De ser cierto que el buen sentido está universalmente repartido, no existirán un lugar propio ni una tarea reservada tan sólo para los filósofos. Al menos no para los filósofos que se ocupan de temas de Bioética.

a) Existen algunos ejemplos de esta percepción difusa de la reflexión filosófica en el campo de la Bioética. Uno de los más significativos es el modo en que se ha regulado hasta ahora en España la composición de los Comités de Ética, por parte de las Administraciones central y autonómicas. Así, la Orden de 1993, vigente en Cataluña, no se refiere en ningún momento a los especialistas en Filosofía, sino a aquella «persona ajena a la institución, con interés acreditado en el campo de la ética» (art. 2, Orden de 14 de diciembre de 1993). En el País Vasco, el Decreto que regula los Comités dice textualmente: «Una persona con acreditada formación en bioética. Un representante de la sociedad con experiencia y prestigio en defensa de los derechos de las personas» (art. 6, Decreto de 7 de febrero de 1995). De forma similar, la Circular del INSALUD sobre este asunto tan sólo menciona la presencia en los Comités de «1 ó 2 personas ajenas a la institución, no vinculadas a las profesiones sanitarias e interesadas en los asuntos bioéticos» (apartado 6, Circular núm. 3, 1995).

b) $\mathrm{Al}$ mismo tiempo, en revistas especializadas e incluso en los medios de comunicación, aparecen con frecuencia los términos «ético»y «bioético», a fin de referirse a especialistas con una metodología y con tareas bien definidas. Se denomina «ético» a aquel profesional que no es médi- co, si bien que está en situación de analizar y de estructurar los temas de Ética médica. El experto se ocupa de cuestiones morales relevantes en distintos campos, máxime si éstas aparecen en situaciones difíciles, complejas. Este especialista aportará claridad, «un mapa de los temas, mostrando caminos, relaciones, funciones, atajos...» ${ }^{8}$. Llevará los problemas a la superficie, los planteará de forma inteligible. Pero nunca tomará las decisiones. En este punto coinciden varios autores, tal como sucede con K. D. Clouser. Este mismo autor ha recordado también que, al comienzo, la mayor parte de los «bioéticos» ${ }^{9}$ eran casi siempre teólogos. Por tal razón se mostraban contrarios al aborto y a otras prácticas que pusieran en cuestión el valor intrínseco de la vida. Poco a poco los expertos fueron asumiendo otra ideología, de tipo secular, adoptando también un estilo más neutro o académico. A. R. Jonsen ${ }^{10}$ explicaba algo parecido, la evolución de un grupo de teólogos, médicos y profesores, que pusieron las bases de una nueva disciplina. ¿Y después? En contextos avanzados, las funciones de los bioéticos están cada vez mejor definidas: éstos realizan informes, atienden consultas, se encargan de actividades formativas, de la investigación y de distintas modalidades de asesoramiento ${ }^{11}$. Al servicio de los profesionales de la salud y de las instituciones. Identifican dilemas, insisten sobre la autonomía moral, influyen en las decisiones de los profesionales... Con todo, nunca dirán lo que ha de hacerse en cada situación.

c) Al examinar más de cerca el papel atribuido a este tipo de expertos, se puede apreciar un desfase poco significativo entre la demanda real de «bioéticos» $\mathrm{y}$, de otro lado, el «buen sentido moral» o universal competencia en temas éticos. Incluso en aquellos casos en que el experto, o asesor moral, ha sido valorado de forma muy positiva ${ }^{12}$, incluso cuando éste ha sido reconocido como una figura necesaria dentro de los comités, en las instituciones, en el 
sistema sanitario o en el sistema educativo, de todas formas se le atribuyen casi siempre tareas de apoyo. Estas actividades tienen que ver más con la comunicación, la práctica argumentativa, la formación de un punto de vista, etc., que con el proceso final: toma de decisiones e intervención directa en los casos reales. Conocido también como «biofilósofo» ${ }^{13}$, este especialista nunca ofrecerá juicios morales particulares. Ni resolverá los problemas concretos que surgen en la práctica médica, ni en el curso de la investigación científica. Sus funciones son otras, éstas consisten básicamente en analizar la vertiente moral de esos problemas, aportando información relevante y una cultivada sensibilidad. $\mathrm{Ha}$ de colaborar con otros expertos e instituciones. Este tipo de experto resulta cada vez más necesario, habida cuenta del aumento de las posibilidades técnicas que modifican en manera sustancial las ideas tradicionales sobre la vida y sobre la muerte. Pero el perfil del especialista es bastante modesto.

Lo anterior sugiere que las habilidades mejor valoradas en el campo de la Bioética - prestar ayuda en los casos prácticos, poner a disposición de otros profesionales una información especializada, formular argumentos específicos, identificar la perspectiva moral, evaluar las actuaciones, uso apropiado del lenguaje moral, etc.requieren un buen conocimiento de métodos y de teorías. Ahora bien, estas habilidades pueden ser atribuidas a profesionales de la Medicina, de la Biología, del Derecho, de las Ciencias Sociales, de las Ciencias de la comunicación. E incluso de la Filosofía... Bastará con que los interesados conozcan en modo adecuado aquellas cuestiones de Ética que surgen en un campo determinado. En el caso de los filósofos, su aportación se ciñe a la deliberación, el análisis, la reflexión. Asunto bien distinto será la toma de decisiones en situaciones reales, por parte de agentes reales. Debido a todo ello, habría que recordar la terminología utilizada en su momento por I. Kant: de una parte, está el «experto el Filosofía práctica»y, por otra parte, el «filósofo práctico». Esta tipología serviría ahora para evitar algunos malentendidos sobre lo que realmente hacen los especialistas en Filosofía moral, en Ética, en Bioética. Sobre todo si se compara luego con las expectativas que ha suscitado su trabajo en los medios de comunicación:

«Un experto en Filosofía práctica no es por eso un Filosofo práctico. Este último es aquel que pone el fin final de razón como principio de sus acciones, al mismo tiempo conecta el saber necesario para ello» ${ }^{14}$.

\subsection{Competencia epistémica}

La terminología kantiana constituye un antecedente de las dos maneras de presentar a los expertos en Ética: éstos tienen un perfil «fuerte» o, por el contrario, un perfil más «débil» o más difuminado. El grado de aplicabilidad de la información condicionará la posición del experto, fuerte o débil. Es decir, algunas ideas previas - sobre el carácter y sobre las tareas que le corresponden- influirán de manera directa sobre el papel asignado al experto. Sus competencias se limitarán al ámbito del conocimiento, la competencia «epistémica». Podrá tener pretensiones más amplias, la competencia «práctica». Sin embargo, la descripción de quién es, qué hace y hasta dónde puede llegar tal experto está mucho más relacionada de lo que parece con las discusiones teóricas. Está relacionada, por ejemplo, con la idea general sobre la Bioética, con la manera de entender la aportación de las éticas aplicadas, el lugar que debe ocupar la Ética como disciplina filosófica, etc. En cierto modo, la figura del experto remite a cuestiones menos inmediatas, cuestiones de Metaética. Puesto que el lugar y el perfil del experto obliga a revisar las expectativas 
generadas en torno a las éticas aplicadas. En sus comienzos, a finales de los años sesenta y principios de los setenta, las éticas especiales dieron la impresión de que, al fin, se había llegado a la situación anunciada en las Tesis sobre Feuerbach, de K. Marx: «Los filósofos no han hecho más que interpretar de diversos modos el mundo, pero de lo que se trata es de transformarlo». Pero era una impresión bastante inexacta. Al menos en lo que se refiere al «especialista moral».

Los términos utilizados en su momento por Kant, experto en Filosofía práctica y Filosofo práctico, subrayaban la distancia entre quien reflexiona y, en otro lado, quien marca de forma directa los objetivos de la acción. En modo análogo, podemos hoy hablar de dos figuras con pretensiones dispares: un «experto práctico» y un «experto en Filosofía práctica». Se atribuirá al primero un saber técnico, similar al que tienen otros especialistas, con dominio de ciertas reglas; el segundo aportará su conocimiento de las tradiciones, los autores, los conceptos y los temas de la Filosofía práctica. Con una influencia limitada sobre los agentes:

1) Es obvio que el perfil más fuerte corresponderá al «experto práctico». Éste tiene una información adecuada, al mismo nivel técnico que reclaman otros profesionales. Su grado de especialización le permite articular, e incluso resolver, aquellos problemas prácticos a los que tenga acceso. Por tanto, ha de conocer y dominar determinadas reglas. En tal sentido, actuará en plano de igualdad con respecto a otros especialistas, participando también en la toma de decisiones sobre los casos que se le presenten. Se le supone, además, la misma competencia moral que tiene cualquier otro agente. La diferencia estriba en que el experto tendrá pretensiones fuertes sobre la aplicabilidad, el éxito de sus recomendaciones. Los agentes que se hallan en situaciones complicadas atenderán a estas recomendaciones, a fin de llegar a las soluciones más adecuadas para ellos. El experto sabe más, de modo que sabrá también cómo aplicar luego la información.

2) El «experto en Filosofía práctica» presenta rasgos más difusos, bastante más débiles que la figura anterior. Este último tiene competencia moral, como todos los agentes. Se ocupa, además, de las cuestiones morales desde un punto de vista peculiar: los considera desde una perspectiva no comprometida, en tercera persona. Descubre dónde se encuentra y en qué consiste la vertiente moral; la analiza, la sistematiza, la discute, la explica a otros, etc. Pero este experto conoce las situaciones a través de autores, textos, teorías: conoce de manera indirecta. Tiene, sin duda, un papel central en los procesos de deliberación, en la argumentación práctica; ahora bien, él se limita a desplegar una actividad reflexiva. No hace recomendaciones, así que influye de forma indirecta, sólo en forma indirecta, en la toma de decisiones. Puesto que las situaciones son singulares, especiales. Los agentes que las protagonizan —en primera línea- perciben los matices e implicaciones mejor que cualquier observador, por imparcial que éste sea. En consecuencia, el experto tendrá una pretensión limitada sobre la aplicabilidad de las teorías que tan bien conoce. Sus objetivos han de ser principalmente la coherencia de los argumentos y la validez de los principios morales, no la respuesta que exigen los casos prácticos singulares.

\section{2. ¿Competencia práctica?}

«Experto epistémico» y «experto preformativo» son considerados de manera muy diferente, según B. Weinstein ${ }^{15}$. La actividad de describir, analizar, justificar e, incluso, la de enseñar todo lo relacionado con la Ética requiere, sin duda, de ciertas 
habilidades. El modo concreto de ejercerlas contribuirá a ensanchar el espacio que ya ocupa la Ética aplicada. Sin embargo, el experto será estimado sobre todo por su conocimiento de los temas y de los argumentos más significativos. Por eso, su contribución al debate práctico tendrá siempre un límite: el experto no está capacitado para intervenir directamente, para «aplicar» reglas generales a situaciones concretas. La distancia entre saber hacer y hacer realmente algo no es del todo insalvable, pero el «experto ético»-B. Weinstein recoge esta terminología- es valorado principalmente por su disciplina intelectual. No por su habilidad para aplicarla. Por estos motivos, él nunca tendrá la consideración reservada a los «expertos por lo que hacen». La capacidad de aplicar las reglas, la performatividad, funciona gracias a un conocimiento previo, a la correspondiente disciplina intelectual. No obstante, las instituciones, comités, servicios de salud, etc., tienden a distinguir claramente entre dos tipos de expertos: aquellos que conocen sobre algo y aquellos que hacen algo.

(i) La separación entre «lo que se conoce» y «lo que se hace» responde a una visión bastante convencional de las relaciones existente entre la teoría y la práctica. El saber y su aplicación no están, en principio, tan alejados entre sí como parece. A pesar de lo cual, la competencia práctica plena sólo será reconocida a quienes hayan sido autorizados para ejercerla. Las instituciones tratan de ser firmes a este aspecto; los códigos de Ética y reglamentos profesionales sirven, entre otras cosas, para establecer un perfil bien definido, quién está autorizado y quién no, en qué condiciones, etc. Pues el dominio de reglas técnicas no garantiza la competencia práctica, y mucho menos la competencia moral. Ésta ha de ser desarrollada por los mismos agentes. En fin, hay por lo menos dos argumentos para reducir las aspiraciones del profesional, sea cual sea su formación: la tendencia a la especialización del conocimiento y, ante todo, el lugar preeminente que ha de ocupar la autonomía de los agentes morales. La competencia moral no es un campo especial del saber, ni los filósofos pueden reemplazar a los agentes a la hora de tomar decisiones. Su campo propio es la reflexión, la deliberación moral, el conocimiento de los conceptos y de las tradiciones. De todo ello se desprende que el experto en Filosofía moral se apoya principalmente en su competencia epistémica. En cambio, no está en situación de reclamar una competencia práctica especial.

(ii) ¿Un perfil débil? Tal vez sea el perfil adecuado a la presente situación. Los avances realizados en la Ética médica, y la trayectoria de la Bioética — del principio de beneficencia al principio de autonomía ${ }^{16}$ - demuestran que los ciudadanos nunca necesitan de expertos que les marquen objetivos para actuar. Ni que prescriban conductas apropiadas a cada caso, tampoco que hagan recomendaciones sobre lo correcto y lo incorrecto, ni que impartan consejos... En definitiva, los ciudadanos son cada vez más conscientes de sus libertades y de su autonomía ${ }^{17}$. La interferencia externa debe ser mínima, en la esfera privada y en la esfera pública. Así pues, el modo de tratar y de resolver los asuntos prácticos ha cambiado de forma sustancial, a medida que las libertades individuales se han ido afianzando en las sociedades avanzadas. La expansión de los derechos ${ }^{18}$ de los pacientes ${ }^{19}$, el consentimiento informado, el rechazo de los errores médicos, los documentos para dejar constancia de las decisiones personales sobre la prolongación de determinados tratamientos médicos, etc., todas estas innovaciones corresponden a la nueva sensibilidad. Ésta consiste en rechazo del paternalismo, actitud consciente, crítica ante las posibilidades abiertas por la ciencia y, ante todo, máximo respeto por las decisiones individuales. 


\section{BIOÉTICA ES ÉTICA}

Parece lógico que los temas relacionados con la esfera privada —el comienzo de la vida, la enfermedad, la muerte - sigan los mismos criterios generales que orientan, o que deben orientar, tanto la acción política como las relaciones internacionales: los Derechos Humanos. Esto es, el esfuerzo por defenderlos a escala global ${ }^{20}$ debe ir en paralelo al esfuerzo por llevarlos en modo efectivo al ámbito de lo personal. Sin embargo, en este ámbito destaca aún más la distancia - antes mencionadaentre lo que corresponde al conocimiento $y$, en otro nivel, la solución de los casos. Dicho de otra manera: las restricciones que pesan sobre el análisis de las cuestiones morales. J. Habermas ${ }^{21}$ ha analizado este antiguo lugar común, la mayor o menor eficacia práctica de las teorías. A diferencia de las respuestas ya ensayadas a lo largo de la Historia de la Filosofía — nada es más práctico que la teoría, según la versión platónica, la teoría se refiere al deber sin que la validez de éste haya de depender del contexto, en la respuesta kantiana; el pensamiento orienta la existencia, en la Filosofía pragmática - este autor subraya el carácter estrictamente normativo de la reflexión moral. Los Derechos Humanos ofrecen el núcleo, el marco adecuado a todo contexto y a toda cultura. Por tal razón, J. Habermas rechaza las alternativas que mantienen un papel de orientador, un papel esotérico para el filósofo. Con lo cual también se desprende de una imagen del filósofo como «experto» entre otros expertos. Consideraremos ahora las razones que sustentan esta versión «débil» de lo práctico. Aunque fuerte por lo que se refiere a la teoría. Versión más extendida en Europa que en Estados Unidos, dicho sea de paso.

1. ¿Cuál ha de ser ahora el papel del intelectual? En las sociedades modernas, cada agente va elaborando su proyecto de vida buena. La pluralidad caracteriza a estas sociedades; por tanto, se trata en principio de sociedades abiertas a distintas formas de entender la existencia. La razón tiene aún una tarea fundamental que cumplir en este contexto, el ejercicio de la crítica. Por otro lado, las teorías tienden hacia la especialización. En consecuencia, el filósofo puede intervenir en la esfera pública, ocupándose de un contenido normativo con validez en los diferentes contextos y en las formas de vida que son diferentes, plurales. Ahora bien, intervendrá de manera distinta a como podrían hacerlo un experto o un terapeuta. Los expertos aportan información especializada, a la manera de los científicos. El filósofo, en cambio, se ocupa de riesgos, de problemas, de cuestiones límite. De un ámbito fronterizo. Sea como fuere, la Filosofía no ofrece lo mismo que otras disciplinas. Ni siquiera está en situación de prestar la ayuda que, en situaciones extremas, promete la Teología. En definitiva, las sociedades complejas han dejado atrás de una idea de filósofo, como alguien capaz de hacer un diagnóstico completo de su tiempo. Por lo demás, tal resultado es coherente con la idea del filósofo como «guarda e intérprete» ${ }^{22}$, que ya había formulado J. Habermas hace algunos años. En medio de una cultura de expertos, el filósofo no desempeña, no puede desempeñar el mismo papel que éstos. Tampoco tiene un ámbito propio, anterior o distinto al de las demás disciplinas. Su tarea consiste principalmente en proteger, defender la racionalidad, gracias al ejercicio de la crítica.

2. La imagen del filósofo como intérprete o mediador, y no como experto - $-\mathrm{y}$ menos aún como «experto moral»—, está relacionada con un estilo de pensar que resulta bastante más común en Europa que en otros contextos. Una tradición concreta, que valora la teoría a la vez que mantiene cierta distancia con respecto a la práctica, el perfil modesto del filósofo moral, todo ello responde a una actitud de cautela. No 
a deficiencias atribuibles a la misma teoría. Vistas así, están más que justificadas las barreras que impiden aplicar, sin más, una idea de la vida, la salud, la muerte, lo correcto, sin exponer las debidas reservas y sin las mediaciones que hagan al caso. La experiencia del control totalitario, los ensayos criminales ${ }^{23}$, los programas de eutanasia que fueron, de hecho, programas de eugenesia — es decir, un pasado infame, no tan lejano- - todo ello gravita aún sobre las versiones más formalistas de la Ética contemporánea. Y sobre las reticencias ${ }^{24}$ alemanas, y europeas, ante el nuevo protagonismo de los expertos en tecnologías de la salud y de la vida. Por lo menos el de aquellos expertos que no ponen su conocimiento y su eficiencia bajo el control de instituciones abiertas y, por tanto, bajo el control de los ciudadanos. Tal prevención ante la «aplicabilidad» o ante las decisiones que parecen opacas, que no se someten a restricciones, deriva sin duda de un precedente trágico. La comunidad científica intervino activamente ${ }^{25}$, y no sólo compartiendo los prejuicios raciales. La figura del «espectador cosmopolita» - como lo denomina R. Rorty ${ }^{26}$ - se ha formado gracias también a la reflexión sobre aquellos hechos. El recuerdo de lo ocurrido sigue pesando hoy sobre el uso de técnicas que, un día, permitirán liberar a la especie humana de graves enfermedades ${ }^{27}$. Pero iexiste, debe existir un límite para la investigación y para la puesta en marcha de las nuevas técnicas? En el año 2001 y a raíz del debate que se produjo en ese país en torno a la clonación de seres humanos, el presidente alemán, J. Rau ${ }^{28}$, volvía a hacerse algunas preguntas clave. Preguntas sobre los límites de la ciencia y sobre la medida de lo humano.

3. Las reformas sociales concretas y las prácticas reales han tenido mayor influencia en otros contextos, como ha explicado R. Rorty ${ }^{29}$. Las ideas sobre lo práctico y, en general, la valoración de las técnicas han tenido un desarrollo indepen- diente en la cultura norteamericana. Debido a esto, la figura del experto y, por extensión, las aplicaciones de la Ética han tenido una notable aceptación en los Estados Unidos. Al margen de otros factores, su cultura de lo práctico influyó de modo decisivo en el surgimiento de la Bioética, como disciplina con una relativa autonomía. Las éticas especiales se beneficiaron de ese mismo ambiente, del sesgo pragmático del momento. De la «ideología americana» ${ }^{30}$ : valoración de las aplicaciones, posturas menos teóricas, compromiso con la objetividad, reformismo, apertura... Los ejemplos reales son sustantivos en el debate moral; por eso, la Ética tiene mucho que aprender la de la Medicina, observa S. Hauerwas ${ }^{31}$. A propósito de este mismo tema, la valoración de las situaciones concretas, A. R. Jonsen ${ }^{32}$ ha explicado que las tradiciones puritanas y, en general, las corrientes del «moralismo americano» apartaron a la Bioética del fundamentalismo, así como de la rigidez que pesa sobre otros enfoques morales. Y, quizás por eso mismo, también la apartaron del modelo de los principios. La importancia atribuida a los casos, así como la tendencia a plantearlos desde dentro, en sus propios términos, impulsaron en modo considerable el desarrollo de la disciplina. A. R. Jonsen ha subrayado el hecho de que la Bioética incorporó poco a poco una moralidad laica, poco fundamentalista, favoreciendo de este modo el estudio de los casos, la investigación, la deliberación moral, el uso de analogías, etc. En cierto modo, modificó la trayectoria seguida hasta entonces por la Filosofía moral: de las teorías a las situaciones problemáticas ${ }^{33}$. Con tales antecedentes, los profesionales «éticos», en la terminología del autor - se interesaron por las situaciones concretas, antes que por los principios generales. Como criterio general, los principios son sometidos a prueba en cada una de las situaciones. Así se deduce también de un conocido documento, el Belmont Report ${ }^{34}$ : los principios constitu- 
yen un marco apropiado para la solución de problemas éticos. Lejos de principios y de valores absolutos, la moralidad se ha convertido, en fin, en el objetivo de nuevas y variadas disciplinas, las «éticas aplicadas» o éticas especiales. El acento estaba y está todavía en la «aplicabilidad» ${ }^{35}$.

4. El interés por lo práctico se ha extendido en un breve lapso de tiempo a otras culturas ${ }^{36}$. También la actitud ambivalente hacia el avance de la biotecnología, que estaba en el origen de la disciplina, la Bioética ${ }^{37}$. Hoy resulta difícil valorar aquel entusiasmo inicial por las éticas especiales, que han proliferado desde los años setenta. Tal vez surgieron de un malentendido - como proponía A. MacIntyre ${ }^{38}$-, puesto que las reglas de conducta no están tan separadas, en el fondo, de su aplicación posterior. Habrá que reconocer, sin embargo, que este malentendido fue muy productivo para la disciplina. El crecimiento de las actividades, interés por los casos, nuevos códigos de Ética profesional, disciplinas especiales, etc., estas novedades crearon un ambiente favorable hacia la figura del «bioético» o experto en cuestiones morales. No sólo porque sus conocimientos fueron requeridos desde el campo de la Medicina, la Biología, la investigación científica, la práctica profesional, etc. También porque la deliberación sobre asuntos de moral se convirtió en «macroética» —así la denomina A. Edel ${ }^{39}$, para diferenciarla de la reflexión que realiza el individuo-; es decir, algo que afectaba a las instituciones y a los ciudadanos. En fin, esta expansión de la Ética, hacia la especialización y hacia la esfera pública, creó las bases para un «giro» de la Filosofía moral, que aún sigue abierto ${ }^{40}$. Y creó también las condiciones para la institucionalización ${ }^{41}$, la profesionalización de los «éticos», «biofilósofos», «bioéticos». ¿Eran realmente necesarios? Hoy no estamos en condiciones de valorar lo sucedido, pero sí en condiciones de examinar más despacio aquel giro hacia lo práctico, así como las expectativas que la Ética y los expertos crearon en aquel momento. Éstos no se limitaron a cruzar ámbitos, a traducir lenguajes, a interpretar culturas. Reclamaron un papel y un espacio propios, como especialistas o «bioéticos». De forma análoga a como se fue afirmando la Bioética ${ }^{42}$ como disciplina especializada.

¿Se trataba de otro malentendido? La cuestión dependerá de hasta dónde llegue la independencia teórica e ideológica de las éticas aplicadas. Valga como ejemplo de esto la postura defendida por A. R. Jonsen, a favor de la «consulta» ${ }^{43}$ ética, y a favor de la figura de los «bioéticos» ${ }^{44}$ : aquellos que se dedican a la Ética clínica. Este autor es decidido partidario de la Nueva Casuística, entendida como discusión de casos particulares, análisis de problemas, de argumentos. Por su método y por su enfoque práctico, la opción de A. R. Jonsen ejemplifica las ventajas, e inconvenientes, asociadas a la independencia teórica. La principal ventaja reside en la flexibilidad, asociada a la tesis de que la Bioética es un «discurso». ¿Cuáles son los inconvenientes? En primer término, la flexibilidad puede quedar reducida a cierta imprecisión, e incluso a debilidad teórica. Y la teoría sigue siendo decisiva. Tras varias décadas de debate y aportaciones del «giro» práctico, se puede constatar que las éticas especiales no han desbancado a la Ética como disciplina filosófica, a pesar del protagonismo que han ido adquiriendo los $\operatorname{casos}^{45}$. En parte, porque la aplicabilidad $^{46}$ no es lo opuesto a la teoría, su alternativa, sino la adecuación de ésta a determinadas situaciones ${ }^{47}$. Y porque la Ética, en tanto disciplina, salvaguarda una visión crítica $^{48}$ de las prácticas y de las aplicaciones especiales. Incluso autores que, como K. D. Clouser ${ }^{49}$, contribuyeron en forma apreciable al desarrollo de la Bioética, han admitido desde el primer momento que de la Ética proceden los principios básicos y los conceptos. Por todos estos motivos, siguen en pie las preguntas sobre 
las relaciones entre la teoría ética y los campos de aplicación. Así, por ejemplo, ¿era necesario desgajar las éticas especiales de la Ética general? ¿Por qué habían de ser disciplinas independientes? Estos temas inciden luego sobre el papel atribuido al experto ${ }^{50}$, al «ético», «bioético», etc. Para decirlo brevemente, esta figura tiene sentido si se está a favor de la Bioética como disciplina independiente con respecto a la Filosofía moral. Por el contrario, en un modelo ${ }^{51}$ más integrado, el debate especializado será exactamente eso, un discurso - la Bioética-y, además, un discurso compartido desde varios puntos de vista y disciplinas.

En segundo lugar, la Bioética surgió en una encrucijada singular: entre la «revolución biológica» ${ }^{52}$ y la emergencia de la contracultura, los movimientos sociales, la crítica radical. Pretendía tender puentes entre ámbitos y entre lenguajes distantes. Y servía para lanzar una mirada crítica sobre las nuevas posibilidades de la ciencia; pero no llevó esta postura hasta su expresión más radical ${ }^{53}$. Más tarde, la disciplina cobró un protagonismo superior al esperado por quienes la habían promovido. En el sentido de que la Bioética dio la impresión de estar al margen de debates teóricos, y de compromisos ideológicos. No fue realmente así: pocos espacios como éste se prestaban tanto a la mezcla de información, discusión racional, valores, visión del mundo, símbolos, emociones ${ }^{54}$. Ni siquiera el análisis estricto, de carácter metaético, había estado al margen de tales compromisos morales, y políticos. En principio, porque las éticas especiales no eran, ni son neutrales desde el punto de vista normativo ${ }^{55}$. Concluyendo, las éticas aplicadas y la Bioética fueron tanto o más sensibles que la Teoría filosófica a los cambios sociales y políticos del momento. Y éstos fueron numerosos, de gran impacto en la opinión pública: contracultura, reacción conservadora, nueva definición de lo privado y de lo público... ¿Cómo olvidar la influencia ejercida por los grupos ${ }^{56}$ y por los movimientos sociales sobre ideas, actitudes, creencias? ¿Qué grupos e instituciones apoyaron en modo significativo la nueva investigación en Bioética? ¿Qué revistas y publicaciones difundieron el nuevo lenguaje y los nuevos proyectos? En 1971, la Universidad de Georgetown acogía un nuevo centro de investigación. El centro contó con la ayuda de la Fundación J. and R. Kennedy ${ }^{57}$.

\section{EXPERTOS Y CIUDADANOS}

En el año 2000, el Informe del Grupo Europeo de Ética —solicitado por el presidente R. Prodi ${ }^{58}$ - se centraba en los recientes desafíos. Desafíos planteados por la biotecnología y por las tecnologías de la información. El documento dejaba asimismo claro el enfoque idóneo para considerar tales asuntos: la primacía de los derechos ciudadanos. La prohibición de prácticas de eugenesia, el respeto por el consentimiento informado, rechazo de la clonación, en contra de la discriminación motivada por las características genéticas o por las condiciones de salud, han sido el resultado de la opción por las libertades y por la dignidad personal. El Informe ponía asimismo énfasis en la Ética y en ciertos valores - pluralismo, tolerancia, dialogo abierto-, que todavía resultan esenciales para la cultura europea. Los principios así establecidos sirvieron para elaborar otro documento de gran importancia, la Carta de los Derechos Fundamentales de la Unión Europea, vigente desde finales del año 2000. Este texto se refiere a los derechos en el campo de la Medicina y de la Biología ${ }^{59}$. Con este marco normativo, los distintos países de la Unión han de desarrollar sus programas de ciencia y tecnología. El Informe y la Carta introducían estos temas de forma similar a como se referían a la igualdad de los ciudadanos y, en general, al ejercicio de los derechos fundamentales. 
Es decir, las cuestiones de Bioética tienen un tratamiento parecido o idéntico al que reciben otros temas de interés para los ciudadanos. Documentos anteriores, como la Resolución de $1996^{60}$, sobre la aplicación de la Biología y la Medicina, así como la Resolución de $1998^{61}$, sobre clonación de seres humanos, insistían ya sobre la protección de la dignidad y de los derechos. Lo mismo cabe decir del Convenio relativo a los derechos humanos y la Biomedicina ${ }^{62}$, suscrito por el Consejo de Europa en 1997. Estos documentos confirman que los temas de Bioética están cada vez más presentes en la esfera pública: el debate europeo está claramente planteado desde una Ética de y para los ciudadanos.

Democracia también para la Bioética, si así puede decirse. Lo cual significa, por ejemplo, control de la Biotecnología a través de la información y el debate públi$\cos { }^{63}$. Este enfoque -lo técnico es también un asunto político, los especialistas han de atender a los intereses y necesidades de los ciudadanos-influye asimismo sobre el grado de aceptación que encuentren los expertos entre los no expertos. Campos especiales, argumentos especiales, información elaborada por quienes conocen la materia, debate abierto, casos de gran complejidad... Hay que admitir que las éticas aplicadas, y la Bioética en particular, se han beneficiado del progreso cuantitativo y cualitativo de la investigación en la ciencia. Junto a esto se debe reconocer otro aspecto: el desarrollo teórico y las aplicaciones prácticas han avanzado gracias a las demandas de los ciudadanos, al apoyo de las instituciones, a decisiones administrativas, a políticas públicas, a la influencia de la opinión ciudadana. Es decir, la Bioética ha ido al ritmo de factores internos y, a la vez, al ritmo marcado por factores externos. De tales circunstancias ha surgido una disciplina de carácter multidisciplinario, no podía ser de otra manera. Sin embargo, estas mismas circunstancias no han reforzado el papel social de los expertos en la materia. Tal vez no debería siquiera plantearse un estatuto singular para éstos. El argumento sostenido aquí es que la disciplina exige conocimientos especializados, no cabe duda. Pero las decisiones sobre el uso del conocimiento no pertenecen a un grupo en especial. Un grupo de perfil difuso, por tanto. Este resultado no acaba, como es obvio, con el desajuste señalado al comienzo: entre las expectativas que rodean a la Bioética y, de otro lado, el papel borroso de los «expertos» en Bioética, en Ética aplicada. A cambio, este enfoque «cívico» refuerza la participación de los no expertos en las decisiones. En la medida en que éstas les afectan.
${ }^{1}$ G. Grass, Mi siglo, Madrid, Alfaguara, 1999, p. 415

${ }^{2}$ M. L. Tina Stevens se refiere al bioético como «especialista moral», «Preface», Bioethics in America, Baltimore, The J. Hopkins University Press, 2000, pp. IX-XIII.

${ }^{3}$ Potter van Rensselaer, Bioethics Bridge to Future, Englewood Cliffs, Prentice Hall, 1971, pp. VII-X.

${ }^{4}$ Décadas más tarde, W. Th. Reich ha explicado cómo fueron los inicios de la Bioética, puente entre ámbitos del saber. La terminología y contenidos de la disciplina fueron esbozados casi al mismo tiempo por dos autores, que trabajaban en centros distintos, y que vieron también el futuro de la Bioética de forma ligeramente distinta: Hellegers y Van Rensselaer Potter, «The Word «Bioethics»: Its Birth and the Legacies of Those Who Shaped It», Kennedy Institute of Ethics Journal, núm. 4, 1994, pp. 319-335. Una visión de conjunto sobre el período formativo de la Bioética se encuentra también en el artículo de R. Gillon, «Bioethics, Overview», en R. Chadwick, Encyclopedia of Applied Ethics, San Diego, Academics Press, 1998, pp. 305-317. 
${ }^{5}$ A. R. Jonsen, «Preface», The Birth of Bioethics, Nueva York, Oxford University Press, 1998, pp. VII-XIV.

${ }^{6}$ R. Descartes, Discours de la méthode, en Oeuvres philosophiques, tomo I, París, Garnier, 1963, p. 368.

7 «Trosième partie», Discours de la méthode, pp. 591-601.

${ }^{8}$ K. D. Clouser, «Medical Ethics: Some Uses, Abuses, and Limitations», The New England Journal of Medicine, núm. 293, 1975, pp. 384-387.

${ }^{9}$ K. D. Clouser, «Bioethics and the Culture Wars», The Nation, 14 de abril de 1997, pp. 23-24.

${ }_{10}$ A. R. Jonsen, «Great Issue of Conscience: Medical Ethics Before Bioethics», The Birth of Bioethics, pp. 3-33.

${ }^{11}$ K. D. Clouser, «What Is A Bioethicist?», The Bioethics Initiative of SouthWestern Ontario, 2001, pp. 1-2, http:// www.thebioethicswebsite.ca

${ }^{12}$ Sobre el papel de asesor o consultor en temas de Ética que preocupan a los profesionales de la salud, «Ethics in Medicine», University of Washington School of Medicine, http:// eduserv.hscer.washington.edu

${ }_{13}$ R. Holmes insistía sobre lo valioso de las técnicas de análisis, que puede ofrecer el experto en la materia o «biofilósofo», «The Limited Relevance of Analytical Ethics to the Problems of Bioethics», Journal of Medicine and Philosophy, núm. 15, 1990, pp. 143-159.

${ }^{14}$ I. Kant, "Vorrede», Die Metaphysik der Sitten, en Werke, Frankfurt, Suhrkamp, 1956, VIII, 2, p. 503.

${ }^{15}$ B. Weinstein, «The Possibility of Ethical Expertise», Theoretical Medicine, núm. 15, 1994, pp. 61-75.

${ }_{16}$ Para el conflicto entre el paternalismo y la autonomía de los pacientes, J. F. Childress, «Paternalism and Autonomy in Medical Decisionmaking», en V. Abernethy, Frontiers in Medical Ethics, Cambridge, Bollinger, 1980, pp. 27-41.

${ }^{17} \mathrm{M}$. Charlesworth ha insistido sobre el lugar que ha de ocupar la autonomía, en el contexto de sistemas democráticos y liberales, "Autonomy and the Liberal Ideal», Bioethics in a Liberal Society, Cambridge, Cambridge University Press, 1993, pp. 10-29.

18 Para la idea de los «derechos como triunfos», R. Dworkin, Taking Rights Seriously, Londres, Duckworth, 1977, p. 367.

${ }^{19}$ La Ley General de Sanidad española, de 1986, recoge los derechos del paciente: respeto a la intimidad, información sobre los servicios, confidencialidad de la información (art. 10).

${ }^{20}$ Sobre la situación de los Derechos Humanos a escala global, J. Habermas, J. «Von der Machtpolitk zur Weltbürgergesellschaft», Zeit der Übergänge, Frankfurt, Suhrkamp, 2001, pp. 27-39.

${ }^{21}$ J. Habermas, «Noch einmal: Zum Verhältnis von Theorie und Praxis», Wahrheit und Rechtfertigung, Frankfurt, Suhrkamp, 1999, pp. 319-333.

22 «Die Philosophie als Platzhalter und Interpret», Moralbewusstsein und kommunikatives Handeln, Frankfurt, Suhrkamp, 1983, pp. 9-30.
${ }^{23}$ Los experimentos médicos y la conducta criminal de algunos científicos durante el Nacionalsocialismo, así como la influencia de aquellos hechos trágicos sobre la posterior idea reducida de «aplicabilidad», temas que han sido analizados, entre otros, por L. Poliakov, «Human Morality and the Nazi Terror», Commentary, núm. 10, 1950, pp. 111-116.

${ }^{24} \mathrm{R}$. Gillon ha insistido sobre las reticencias que suscitó la Bioética en Alemania, «Bioethics, Overview», en R. Chadwick, Encyclopedia of Applied Ethics, pp. 305-317.

25 P. Steinfels, «Introduction», en P. Steinfels, Biomedical Ethics and the Shadow of Nazism, Hastings Center, Special Supplement, 1976, pp. 1-2.

${ }_{26}$ R. Rorty, Forjar nuestro país, Barcelona, Paidós, 1999, p. 95.

27 J. Albrecht consideraba las diferencias entre «eugenesia negativa» $\mathrm{y}$ «eugenesia positiva», «Die Guten ins Tröpfchen», Die Zeit, núm. 38, 1999, p. 16.

${ }_{28}$ Discurso del 20 de mayo de 2001, J. Rau, «Wird alles gut?- Für einem Fortschritt nach menschlichen Mass», Berliner Rede, Staatsbibliotek zu Berlin.

${ }^{29}$ R. Rorty, Forjar nuestro país, pp. 93-95.

${ }^{30}$ Los elementos de la ideología americana fueron examinados por H. T. Wilson, The American Ideology, Londres, Routledge, 1977, pp. 11-28.

${ }^{31}$ S. Hauerwas, «Preface», Suffering Presence, Notre Dame, University of Notre Dame Press, 1986, pp. IX-X.

${ }^{32}$ A. R. Jonsen, «American Moralism and the Origin of Bioethics in the United States», Journal of Medicine and Philosophy, núm. 16, 1991, pp. 115-129.

${ }^{33}$ A. R. Jonsen, "Casuistry as Methodology in Clinical Ethics», Theoretical Medicine, núm. 12, 1991, pp. 295-307.

${ }^{34}$ The National Commission for the Protection of Human Subjects of Biomedical and Behavioural Research, The Belmont Report, 18 de abril de 1979.

${ }_{35}$ M. D. Bayles, «Ethical Theory in the Twenty-First Century», en J. P. de Marco y R. M. Fox, New Directions in Ethics, Londres, Routledge a. Kegan Paul, 1986, pp. 249-264.

${ }^{36} \mathrm{D}$. Gracia ha presentado una visión de conjunto sobre del origen, desarrollo, la situación actual de la Ética médica y la Bioética en España, «Vent'anni di Bioética nell'area linguística spagnola», en C. Viafora, Vent'anni di Bioética, Padua, Lanza, 1990, pp. 251-299.

${ }_{37}$ M. L. Tina Stevens ha llamado la atención sobre la actitud ambivalente hacia el progreso científico, uno de los elementos decisivos en la formación de la disciplina, «Prologue. The Tradition of Ambivalence», Bioethics in America, pp. 1-7.

38 A. MacIntyre, «Does Applied Ethics Rest on a Mistake?», The Monist, núm. 67, 1984, pp. 498-513.

39 A. Edel diferencia entre la «microética», dilemas del individuo, y la «macroética», cuestiones que se tratan en el marco institucional; «Ethical Theory and Moral Practice: On the Terms of Their Relation», en J. P. de Marco y R. M. Fox, New Directions in Ethics, pp. 317-335. 
40 Para un comentario sobre el «giro aplicado», J. M. ${ }^{a}$ García Gomez-Heras, «El retorno a una ética de la vida cotidiana», en J. M. ${ }^{a}$ García Gomez-Heras, Dignidad de la vida y manipulación genética, Madrid, Biblioteca Nueva, 2002, pp. 25-39. El significado de la Ética «aplicada» y del «giro aplicado» ha sido examinados por A. Cortina, «El estatuto de la ética aplicada. Hermenéutica crítica de las actividades humanas», Isegoría, núm. 13, 1996, pp. 119-134.

${ }^{41}$ R. C. Fox, «Is Medical Education Asking Too Much of Bioethics?», Daedalus, núm. 128, 1999, pp. 1-25.

${ }^{42}$ La Bioética, como una disciplina con perfil propio, integrada en las actividades y programas académicos, ha sido analizada por S. Gorowitz, «Bioethics», en L. C. Becker y Ch. B. Becker, Encyclopedia of Ethics, Nueva York, Garland, 1992, pp. 89-91.

${ }^{43}$ A. R. Jonsen, «Can An Ethicist Be A Consultant?», en V. Abernethy, Frontiers in Medical Ethics, pp. $157-172$.

${ }^{44}$ A. R. Jonsen, «Casuistry as Methodology in Clinical Ethics», Theoretical Medicine, núm. 12, 1991, pp. 295-307.

${ }^{45}$ Para un análisis de los dilemas prácticos desde un modelo de principios, M. T. López de la Vieja, Principios y casos prácticos, Madrid, Tecnos, 2000, pp. 17-35.

${ }^{46} \mathrm{~J}$. Habermas la define como el proceso de elegir una norma a luz de una situación dada, «Richtigkeit vs. Wahrheit», Deutsche Zeitschrift für Philosophie, núm. 46, 1998, pp. 179-208.

${ }^{47}$ M. T. López de la Vieja, «Relevancia y aplicabilidad en Ética», Doxa, núm. 20, 1997, pp. 427-440.

${ }^{48}$ Para la conexión entre parte teórica y vertiente aplicada de la Ética, E. Morscher, O. Neumeier y P. Simons, «Introduction: On Apllying Ethics», Applied Ethics in a Troubled World, Dordrecht, Kluwer, 1998, pp. IX-XXI.

${ }^{49}$ K. D. Clouser, «Biomedical Ethics: Some Reflections and Exhortations», The Monist, núm. 60, 1977, pp. 47-61.

${ }^{50}$ V. Camps ha expresado sus reservas en torno a la función del experto, el «bioeticista», «La misión del filósofo: ¿Sócrates o Platón?», Una vida de calidad, Barcelona, Crítica, 2001, pp. 211-229.

${ }^{51}$ Sobre las diferencias entre modelo que propicia la independencia de las éticas especiales y, por otro lado, la integración o dependencia de las disciplinas especiales con respecto a la Ética, M. T. López de la Vieja, «Principios morales en la Ética aplicada», Agora, núm. 16, 1997, pp. 157-166.

${ }_{52}$ M. L. Tina Stevens, «The Culture of Post-atomic Ambivalence», Bioethics in America, pp. 8-45.

${ }_{53}$ M. L. Tina Stevens explica los inicios de la Bioética como resultado de un equilibrio, entre la pretensión de legitimidad para la ciencia y, de otro lado, sus intentos de eludir una crítica externa, «Preface», Bioethics in America, pp. IX-XIII.

${ }^{54}$ I. Kato recordaba que la Bioética se presta habitualmente a la confusión, entre elementos racionales y elementos emotivos, «Why Bioetics Sought In Our Contemporary Society?», ARSP, Bhf.39, 1991, pp. 1-13.

${ }_{55} \mathrm{~W}$. T. Blackstone distinguía entre la neutralidad de las teorías ante la conducta y, luego, la neutralidad normativa, «Are Metaethical Theories Normatively Neutral?», Australasian Journal of Philosophy, núm. 39, 1961, pp. 67-74.

${ }^{56}$ R. Tatalovich y B. W. Daynes insisten sobre la politización de los conflictos morales desde los grupos, la Corte Suprema, las instituciones, etc., «Introduction. Social Regulation and Moral Conflict», en T. Tatalovich y B. W. Daynes, Moral Controversies in American Politics, Nueva York, Sharpe, 1998, pp. XXIX-XXXIV.

57 «Bioethics Center Formed», Chemical \& Engineering, 11 de octubre de 1971, p. 7.

58 Citizens Rights and the New Technologies: A European Challenge, Bruselas Report, 23 de mayo de 2000.

${ }^{59}$ Carta 2000/C 364/01, art. 3.2.

${ }^{60}$ Resolución sobre la protección de los derechos humanos y de la dignidad humana en el marco de la aplicación de la Biología y la Medicina, B4-1029, 1082, 1084 y 1085/96.

${ }^{61}$ Resolución sobre la clonación de seres humanos, B4-0050, 0053, 0068, 0074 y 0087/98.

${ }^{62}$ Convenio para la protección de los derechos humanos y la dignidad del ser humano con respecto a las aplicaciones de la Biología y la Medicina, 1997.

${ }_{63}$ A. Kaufmann es partidario de ese control a través de la discusión pública, comisiones, grupos de trabajo, etc., «Rechtsphilosophische Reflexionen über Biotechnologie und Bioethik an der Schwelle zum dritten Jahrtausend», ARSP, Bhf.39, 1991, pp. 14-33. 